\title{
PERFIL BIOSOCIAL DE LAS USUARIAS QUE DEMANDAN LA PÍLDORA DE ANTICONCEPCIÓN DE EMERGENCIA EN UN CENTRO DE SALUD DE SANTIAGO, 2006-2007
}

\author{
Bárbara Escobar L. ${ }^{1 a}$, Carolina Román J. ${ }^{1 a}$, Carla Muñoz T. ${ }^{1 a}$, Fanny López A. ${ }^{1 b}$ \\ ${ }^{1}$ Escuela de Obstetricia y Puericultura, Facultad de Ciencias Médicas, Universidad de Santiago de Chile.
}

$a_{\text {Licenciada en Obstetricia y Puericultura. }}{ }^{b}$ Matrona.

\section{RESUMEN}

Antecedentes: En Chile aproximadamente 475.297 mujeres se encuentran expuestas al riesgo de embarazos no deseados y abortos provocados, ya sea porque usan anticonceptivos de baja eficacia o no los usan. Objetivos: Conocer el perfil biosocial de las usuarias que demandan la píldora de anticoncepción de emergencia, una vez que ésta estuvo disponible para las beneficiarias de los Consultorios de Atención Primaria del Servicio de Salud. Métodos: Se analizó las características sociodemográficas y antecedentes gineco-obstétricos de la totalidad de las 93 fichas de las mujeres que solicitaron anticoncepción de emergencia entre el segundo semestre de 2006 y el primer semestre de 2007, en un Consultorio de Atención Primaria de Salud del Servicio de Salud Metropolitano de Santiago. Resultados: El perfil obtenido es de una mujer joven de promedio de 23,6 años, soltera, estudiante, beneficiaria exenta de pago del sistema de salud público, con edad promedio de inicio de relaciones sexuales de 16,2 años, que ha tenido entre una y dos parejas sexuales, sin hijos, que no utiliza un método anticonceptivo habitual, que consulta por haber tenido una relación sexual no protegida en las últimas 24 horas. Conclusiones: El perfil identificado corresponde a una población de conducta sexual desprotegida, de alto riesgo de embarazo no deseado y de aborto inseguro, que deben ser incorporadas a los programas regulares de control de la fecundidad.

\section{PALABRAS CLAVES: Anticoncepción de emergencia, perfil de usuaria, Yuzpe}

\section{SUMMARY}

Background: In Chile approximately 475,297 women are in risk of unwanted pregnancies and induced abortions, either because of low effectiveness contraceptive use or not use at all. Objective: To determine the biosocial profile of users who demand the pill of emergency contraception once they were available to the beneficiaries of a primary care health service. Method: This investigation analyzed sociodemographic characteristics and background obstetric-gynecological of 93 medical files of women who requested emergency contraception in a Primary Care Health of the Central Region Metropolitan Santiago, between the second semester of 2006 at first semester of 2007. Results: The profile obtained was a young woman of 23.6 years, single, student, beneficiary exempt of paying in the public health system, with an age of onset sexual intercourse of 16.2 years, who has had between one and two sexual partners, without children, who are not using a regular contraceptive method, and who consults mainly by having unprotected sexual intercourse in the last 24 hours. Conclusions: The profile identified corresponds to a population of unprotected sexual conduct, with a high risk of unwanted pregnancy and unsafe abortion, which should be incorporated into regular programmes of fertility control.

KEY WORDS: Emergency contraception, user profile, Yuzpe 


\section{INTRODUCCIÓN}

El $53,5 \%$ de la población femenina chilena esta en edad fértil, lo que corresponde a 4.100.047 mujeres de 15 a 49 años, según el último censo del año 2002 (1). El 47,5\% de estas mujeres (1.947.522 mujeres) usan métodos anticonceptivos y de estas, un 6,5\% (126.588 mujeres) son de baja eficacia como los métodos de barrera y los naturales (2).

No usan anticonceptivos un $52,5 \%$ de las mujeres (2.152.525 mujeres) y de este grupo, un $83,8 \%$ no los usa porque refiere no necesitarlo, pero lo que es mas relevante el 16,2\% (348.709 mujeres) no los usa y los necesita (2). De lo que se desprende que aproximadamente un total de 475.297 mujeres se encuentran expuestas al riesgo de embarazos no deseados y abortos provocados, ya sea porque usan anticonceptivos de baja eficacia o no los usan.

Satisfacer estas y otras necesidades anticonceptivas son los objetivos de las Normas Nacionales sobre Regulación de la Fertilidad, para lo cual ofrecen una amplia gama de anticonceptivos basado en los criterios médicos de elegibilidad para el uso de anticonceptivos de la Organización Mundial de la Salud, los cuales se ajustan a la evidencia científica más reciente y a los últimos acuerdos internacionales en materia de manejo clínico de la anticoncepción $(3,4)$. Una de las opciones ofrecidas son las píldoras anticonceptivas de emergencia, alternativa para las mujeres que han tenido relaciones sexuales no protegidas o han experimentado una falla en el método anticonceptivo y que no desean quedar embarazadas (5). En Chile, al Estado le corresponde, a través de sus políticas públicas de sexualidad y reproducción, resguardar los derechos de la población, incorporando la diversidad de opciones disponibles, de modo que el abanico de posibilidades sea tan amplio, que incluya cualquier situación de vida, condición de salud y perspectiva valórica (4).

El objetivo de este estudio es conocer el perfil biosocial de las usuarias que demandan anticoncepción de emergencia, cuando fue puesta a disposición de las beneficiarias de un Consultorio de Atención Primaria del Servicio de Salud Metropolitano Central, en el período 2006-2007, para brindar a los responsables de las políticas y de la toma de decisiones, antecedentes que puedan utilizarse en la revisión de las guías nacionales sobre anticoncepción, teniendo en cuenta las consideraciones de conducta de criterios no médicos y sociales.

\section{PACIENTES Y MÉTODOS}

Se estudia la totalidad de las 93 fichas de mu- jeres que solicitaron anticoncepción de emergencia entre el segundo semestre de 2006 y el primer semestre de 2007, en un Consultorio de Atención Primaria de Salud del Servicio de Salud Metropolitano Central de la Región Metropolitana de Santiago. El estudio es de tipo observacional, descriptivo y retrospectivo. Los criterios de inclusión fueron fichas de mujeres que solicitaron anticoncepción de emergencia en el período y consultorio en estudio. Las variables se clasificaron en: a) variables que definen el perfil sociodemográfico de las participantes (edad, estado marital, ocupación y tipo de previsión de salud) y b) variables relacionadas con los antecedentes gineco-obstétricos (edad de inicio de la actividad sexual, número de parejas sexuales, paridad, uso previo de métodos anticonceptivos, causas de solicitud de la anticoncepción de emergencia, régimen de anticoncepción de emergencia entregado, tiempo entre el coito y la ingestión del anticonceptivo de emergencia y número de veces que a usado la anticoncepción de emergencia).

La recolección de datos se realizó desde las fichas de las usuarias a una pauta de cotejo. La tabulación de los datos se realizó en planillas Excel y para el análisis de datos se utilizó estadística descriptiva.

\section{RESULTADOS}

La edad promedio de las mujeres que solicitaron anticoncepción de emergencia fue 23,6 \pm 8,5 años, una moda de 17 años, una mediana de 22 años y un rango de edades extremas de 15 y 47 años.

Más de la mitad $(51,1 \%)$ son mujeres solteras, sin pareja estable, sin trabajo remunerado $(97,9 \%)$ ya que son estudiantes, mujeres que se dedican al cuidado de su hogar o cesantes. La mayoría son beneficiarias exentas de pago del Sistema de Salud Público, adheridas a FONASA A o B (66\%) (Tabla I).

La edad de inicio de las relaciones sexuales fluctuó entre los 13 y 22 años, teniendo como edad promedio $16,2 \pm 2,2$ años y una moda de 15 años. La gran mayoría han tenido entre 102 parejas sexuales a lo largo de su vida (80,6\%) (Tabla II). El $57,4 \%$ de las usuarias de anticoncepción de emergencia son nuligestas, un $4,3 \%$ tuvo solo antecedentes de algún tipo de aborto y el 38,3\% tienen de 1 a 3 partos (Tabla III).

Durante el mes anterior a la solicitud de anticoncepción de emergencia, el 59,6\% de las mujeres no utilizaron ningún método anticonceptivo, las restantes usaron preservativo $(29,8 \%)$, anticonceptivos orales $(8,5 \%)$ y el dispositivo intrauterino $(2,1 \%)$.

La principal causa de solicitud de la anticoncepción de emergencia fue una relación sexual no 
Tabla I

DISTRIBUCIÓN DEL PERFIL SOCIODEMOGRÁFICO DE LAS USUARIAS DE ANTICONCEPCIÓN DE EMERGENCIA

\begin{tabular}{|c|c|c|c|c|c|}
\hline Estado marital & $\%$ & Ocupación & $\%$ & Previsión & $\%$ \\
\hline Casada & 17,0 & Estudiante & 57,5 & Fonasa A & 46,9 \\
\hline Soltera sin pareja estable & 51,1 & Dueña de casa & 34,0 & Fonasa B & 19,1 \\
\hline Conviviente & 6,4 & Con trabajo & 2,1 & Fonasa C & 19,1 \\
\hline Divorciada & 2,1 & Sin trabajo & 6,4 & Fonasa D & 14,9 \\
\hline \multirow[t]{2}{*}{ Pololeando } & 23,4 & - & - & - & - \\
\hline & 100,0 & & 100,0 & & 100,0 \\
\hline
\end{tabular}

Tabla II

DISTRIBUCIÓN DE LAS CARACTERÍSTICAS DE LA ACTIVIDAD SEXUAL DE LAS USUARIAS DE ANTICONCEPCIÓN DE EMERGENCIA

\begin{tabular}{cccr}
\hline $\begin{array}{l}\text { Edad de inicio } \\
\text { de las relaciones } \\
\text { sexuales (años) }\end{array}$ & $\%$ & \multicolumn{3}{c}{$\begin{array}{c}\text { Número de parejas } \\
\text { sexuales durante } \\
\text { su vida }\end{array}$} & $\%$ \\
\hline $12-14$ & 19,1 & 1 & 36,7 \\
$15-17$ & 57,1 & 2 & 43,9 \\
$18-20$ & 21,4 & 3 & 14,6 \\
$21-23$ & 2,4 & 4 & 2,4 \\
- & - & 6 & 2,4 \\
\hline & 100,0 & & 100,0 \\
\hline
\end{tabular}

protegida $(61,7 \%)$, el segundo motivo fue la ruptura del preservativo $(27,6 \%)$, seguido por el olvido de tomar una píldora de anticoncepción oral $(6,4 \%)$ y el abuso sexual $(4,3 \%)$.

Para satisfacer esta solicitud, fue prescrito el régimen llamado Postinor a un $72,3 \%$ de las mujeres solicitantes y el método Yuzpe al 27,7\% de las mujeres. Estas terapias fueron solicitadas en la mayoría de los casos en un tiempo menor a las 24 horas post coito no protegido $(53,2 \%)$ y $40,4 \%$ entre las 24 y 48 horas (Tabla IV). Casi la totalidad de las mujeres $(93,6 \%)$ había requerido este régimen de anticoncepción de emergencia por primera vez.

\section{DISCUSIÓN}

Este estudio determinó que las características
Tabla III

DISTRIBUCIÓN DE LAS MUJERES USUARIAS DE ANTICONCEPCIÓN DE EMERGENCIA SEGÚN ANTECEDENTES GINECOOBSTETRICOS

\begin{tabular}{crcr}
\hline$N^{\circ}$ de gestaciones & $\%$ & $N^{\circ}$ de partos & $\%$ \\
\hline 0 & 57,4 & solo abortos & 4,3 \\
1 & 19,1 & 0 & 57,4 \\
2 & 10,6 & 1 & 14,9 \\
3 & 12,9 & 2 & 10,6 \\
- & - & 3 & 12,8 \\
\hline
\end{tabular}

sociodemográficas de las solicitantes de anticoncepción de emergencia corresponde a una mujer joven, estudiante y soltera, coincidente con las usuarias de este tipo de anticoncepción de otros centros de salud de regiones de América y Europa $(6,7,8,9)$.

En cuanto a la previsión de las usuarias más del $65 \%$ son beneficiarias exentas de pago del sistema de salud público por estar adheridas a FONASA A o B. Lo cual se asemeja con lo referido en la Primera Encuesta Nacional de Calidad de Vida y Salud donde el $53,8 \%$ de las personas encuestadas que usa anticonceptivos refiere que los obtuvo de un servicio de salud público (2), lo cual refuerza que para el uso de anticonceptivos, las mujeres buscan los servicios de salud públicos.

La edad de inicio de las relaciones sexuales fue 
Tabla IV

\section{DISTRIBUCIÓN DE LAS MUJERES USUARIAS DE ANTICONCEPCIÓN DE EMERGENCIA SEGÚN} SUS CARACTERÍSTICAS DE USO

\begin{tabular}{cclc}
\hline $\begin{array}{c}\text { Tiempo (horas) entre el coito y la } \\
\text { ingestión de las PAE }\end{array}$ & $\%$ & $\begin{array}{c}\text { Causas de solicitud } \\
\text { de las PAE }\end{array}$ & $\%$ \\
\hline$<24$ & 53,2 & Coito no protegido & 61,7 \\
$24-48$ & 40,4 & Rotura del preservativo & 27,6 \\
$48-72$ & 4,3 & Olvido de ingestión de ACO & 6,4 \\
$>72$ & 2,1 & Abuso sexual & 4,3 \\
\hline & 100,0 & & 100,0 \\
\hline
\end{tabular}

PAE: píldora de anticoncepción de emergencia. ACO: anticonceptivo oral.

16,2 años, similar a los datos del estudio realizado en un Centro de Planificación Familiar de Madrid, donde las mujeres que solicitaron anticoncepción de emergencia en el año 2000, tenían 18 años como edad promedio de la primera relación sexual (10). Esta edad de inicio, no se diferencia mucho de la prevalencia nacional publicada en la Quinta Encuesta Nacional de Juventud del año 2006, que revelo que la edad de inicio de las relaciones sexuales de la juventud femenina chilena era 17,8 años (11). De ahí que el $80 \%$ de las solicitantes de anticoncepción de emergencia han tenido entre 1 a 2 parejas sexuales, lo que se asemeja con el número promedio de 1,8 parejas sexuales de la población femenina sexualmente activa de nuestro país, según la Encuesta Nacional de Comportamiento Sexual de la Comisión Nacional del SIDA (2000) (12).

En los antecedentes gineco-obstétricos de las mujeres en estudio, se observó que $57,4 \%$ son nuligestas y $4,3 \%$ tienen antecedentes de aborto. En un estudio realizado en población británica se concluyó que las solicitantes de anticoncepción de emergencia tuvieron una asociación de 2,4 de tener antecedentes de aborto en relación al resto de la población (7). Esto es importante, ya que las mujeres chilenas tienen la segunda mayor prevalencia latinoamericanas de tener antecedentes de aborto por embarazo no deseado (4,5\%) (13).

En el mes anterior a la solicitud de anticoncepción de emergencia, un $60 \%$ de las solicitantes no usaron ningún método anticonceptivo, esta baja prevalencia de uso de anticonceptivos coincide con los resultados de la $\mathrm{V}$ Encuesta Nacional de la Juventud (2006), que concluye que las prácticas sexuales de los jóvenes permiten hablar de una conducta sexual desprotegida, al verificar que $57,9 \%$ de las jóvenes no uso métodos anticonceptivos en su primera relación sexual (11). Esta baja prevalencia de anticoncepción de uso regular, dio como resultado que la principal causa de solicitud de la anticoncepción de emergencia fue una relación sexual no protegida $(61,7 \%)$, lo que concuerda con la guía de prestación de la OMS que refiere que la razón principal de demanda de este tipo de anticoncepción son las relaciones sexuales sin protección (14). Este resultado coincide con el estudio realizado en 4 centros de salud de la organización Marie Stopes en Bolivia (15) el año 2006 donde el $85 \%$ de las mujeres que solicitó la anticoncepción de emergencia no usaba ningún método anticonceptivo, pero difiere de dos estudios realizados en España donde el preservativo fue el método más utilizado $(85 \%)$ por las usuarias que solicitaron anticoncepción de emergencia según el estudio realizado por Cárdenas y cols en 2002 (16) y un 88,8\% en el estudio realizado por Vergara y cols en el año 2004 (6).

Para satisfacer la demanda de anticoncepción de emergencia, en el Consultorio en estudio existen dos regimenes: Postinor y Yuzpe. Ambas prescripciones, fueron solicitadas en un $53,2 \%$ de las veces, antes de las 24 horas. En países europeos y norteamericanos un mayor porcentaje de usuarias asisten a los centros de salud más precozmente que en nuestro país, debido a los horarios de atención de los servicios más adecuados a este tipo de demanda $(6,8,16)$.

En relación al número de veces que las usuarias solicitaron la anticoncepción de emergencia, solo un $6,4 \%$ lo había solicitado más de una vez. Esta demanda es diferente a los centros de Europa 
y América, en la cual la población de mujeres reincidentes de esta anticoncepción de emergencia llega hasta un $33 \%$, por lo tanto, el término "píldoras anticonceptivas de emergencia", es el adecuado al transmitir el importante mensaje de que no deben ser utilizadas como un método anticonceptivo regular (6,15-18), enfatizándose la importancia de la consejería pos anticoncepción de emergencia.

\section{CONCLUSIÓN}

La población femenina que solicitó anticoncepción de emergencia se caracterizó por ser joven, soltera, estudiante, sin hijos, pobre, con edad pre$\mathrm{coz}$ de inicio de relaciones sexuales, que ha tenido parejas sexuales previamente y que no utiliza un método anticonceptivo regular. Existiría en esas condiciones para este tipo de anticoncepción una potencial población demandante de aproximadamente 4 millones de personas, que debe ser reducida incorporándolas a programas regulares de control de la fecundidad, para reducir el riesgo de embarazo no deseado y de aborto inseguro.

\section{BIBLIOGRAFÍA}

1. Instituto Nacional de Estadísticas (INE). Censo 2002. Chile: INE, 2002.

2. Ministerio de Salud. Departamento de Epidemiología. Departamento de Promoción de Salud. Primera Encuesta Nacional de Calidad de Vida y Salud 2000. Chile 2001. Santiago, Chile.

3. Organización Mundial de la Salud. Criterios Médicos de elegibilidad para el uso de anticonceptivos. $3^{\mathrm{a}}$ ed. Ginebra, Organización Mundial de la Salud, 2005.

4. Ministerio de Salud de Chile. Normas Nacionales sobre Regulación de la Fertilidad: Normas técnicas y guías clínicas sobre regulación de la fertilidad. Santiago, Chile, 2006.

5. Organización Mundial de la Salud. Recomendaciones sobre prácticas seleccionadas para el uso de anticonceptivos. $2^{\mathrm{a}}$ ed. Ginebra, Organización Mundial de la Salud. 2005.

6. Vergara J, López-Guerrero A, López F. Anticoncepción de emergencia: Perfil de la usuaria en servi- cios de urgencia en atención primaria. Aten Primaria 2004;34(6):279-85.

7. Black $\mathrm{KI}$, Mercer $\mathrm{CH}$, Johnson AM, Wellings $\mathrm{K}$. Sociodemographic and sexual health profile of users of emergency hormonal contraception: Data from a British probability sample survey. Contraception 2006;74(4):309-12.

8. Loughrey F, Matthews A, Bedford D, Howell F. Characteristics of women seeking emergency contraception in general practice. Ir Med J 2006;99(2):50-2.

9. Andrade R, Guimaräes A, Fagotti Filho A, Carvalho N, Arrabal J, Rocha D, Medeiros M. Características demográficas e intervalo para atendimento em mulheres vítimas de violência sexual. Rev Bras Ginecol Obstet 2001;23(9):583-7.

10. Ruiz S, Güell E, Herranz C, Pedraza C. Anticoncepción poscoital: Características de la demanda. Aten Primaria 2002; 30(6):381-7.

11. Instituto Nacional de la Juventud (INJUV). V Encuesta Nacional de Juventud. Chile 2006. Santiago, Chile.

12. Comisión Nacional del SIDA (CONASIDA). Encuesta Nacional de Comportamiento Sexual. Chile 2000. Santiago, Chile.

13. The Alan Guttmacher Institute. Clandestine Abortion: Hallado en: http://www.celsam.org/home/manual. asp?cve_manual=8. Acceso el 20 de Enero de 2008.

14. Organización Mundial de la Salud. Anticoncepción de Emergencia: Guía para prestación de servicios. Ginebra. Organización Mundial de la Salud, 2003.

15. Torres R. Anticoncepción de emergencia en los centros Marie Stopes: Bolivia. 2006;1-3. Disponible: http://www.aoe.org.pe/downloads/PUBLICACIONES/ Anticoncepcion_Emergencia_MarieStopes.pdf. Acceso del 20 de Enero de 2008.

16. Cárdenas D, Parrilla $F$, Mengíbar $M$, Espinosa $M$, Issa Z, Cárdenas A. Perfil de solicitante de la píldora postcoital (Levonorgestrel) en unidades de urgencias. Med Familia 2002;3(3):179-83.

17. Verhoeven V, Peremans L, Avonts D, Van Royen P. The profile of emergency contraception users in a chlamydia prevalence study in primary care in Belgium. Eur J Contracept Reprod Health Care 2006;11(3):17580.

18. Consorcio Internacional sobre Anticoncepción de Emergencia. Píldoras anticonceptivas de emergencia: Guía médica para la prestación de servicios. $2^{\underline{a}}$ ed. Washington, 2003. 